analysis into patients who recovered or died depending on ward based care versus ITU.

Conclusion COVID pneumonitis influenced the outcome of patients admitted with hepatic decompensation resulting in increased mortality. Alcohol was the commonest cause of underlying liver disease in both Pre-COVID and COVID period. Age, nature of hepatic decompensation (Gastrointestinal bleeding, hepatic encephalopathy, spontaneous bacterial peritonitis) CLIF-C score for patients referred to ITU and average hospital stay was not much different in both the groups. The median MELD and ACLF grade were higher in patients who died compared to those who recovered.

\section{P084 A MELD SCORE $\geq 19$ PRIOR TO ANTI-THYMOCYTE GLOBULIN THERAPY FOR STEROID RESISTANT T CELL- MEDIATED REJECTION PREDICTS POOR 5-YEAR TRANSPLANT FREE SURVIVAL}

Lindsay Smith*, Oliver Tavabie, Niloufar Safinia, Cathrine McKenzie, Andreas Prachalias, Krishna Menon, Kosh Agarwal, Michael Heneghan, Varuna Aluvihare. King's College Hospital, UK

\subsection{6/gutjnl-2021-BASL.92}

Background and Aims Steroid resistant $\mathrm{T}$ cell-mediated rejection is a risk factor for graft loss and patient mortality post liver transplant (LT). Anti-thymocyte globulin (ATG) therapy has been demonstrated to be an effective treatment for this condition but long term follow up data are limited. We aimed to identify risk factors for reduced 1-year and 5-year LT free survival post ATG use for steroid resistant $\mathrm{T}$ cell-mediated rejection.

Method We performed a retrospective observational study evaluating all LT patients who received ATG for steroid resistant cellular rejection between 01/2007-01/2020 at King's College Hospital. ATG was administered based on institutional practice and clinician choice. Patients were excluded if they received ATG without prior steroids. Clinical, demographic and laboratory data were collected and univariate analysis were performed.

Results 39 LT patients fulfilled inclusion/exclusion criteria. 13 did not achieve a 1-year LT-free survival and a further 5 did not achieve a 5-year LT free survival. Age, female sex, LT for

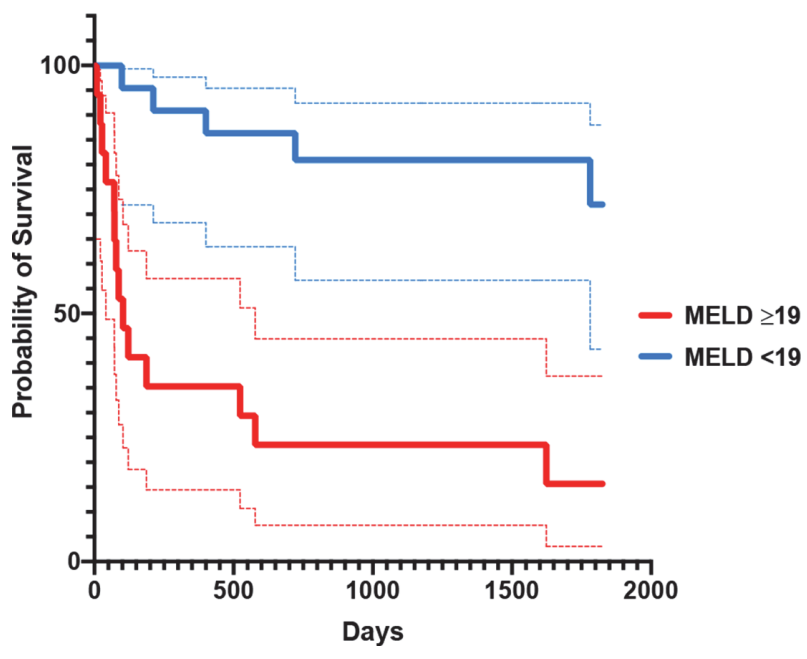

Abstract P084 Figure 1 an autoimmune liver disease, redo LT and pre-ATG AST, tacrolimus level, creatinine, albumin, haemoglobin, white cell count and differentials did not predict 1-year LT free survival. Variables predicting reduced 1-year LT free survival were:increased time from LT when receiving ATG (AUC 0.82, 95\% CI 0.69-0.96), histological overlap with chronic rejection (AUC 0.75 , 95\% CI 0.57-0.93), increased pre-ATG bilirubin (AUC 0.80, 95\% CI 0.66-0.94), INR (AUC 0.71, 95\%CI $0.52-0.89$ ) and MELD (AUC 0.86, 95\% CI 0.75-0.97). Survival analysis was performed to evaluate risk factors affecting 5-year LT free survival. Cut-off values for pre-ATG MELD and days post-LT receiving ATG were determined by Youden's Index. Histological overlap with chronic rejection (HR 3.33, 95\% CI 1.10-10.11), receiving ATG $>178$ days post-LT (HR 5.86, 95\% CI 1.82-18.83) and a pre-ATG MELD $\geq 19$ (HR 8.84, 95\% CI 3.25-24.04) (see figure 1) predicted a reduced 5 -year survival.

Conclusion ATG use in patients with steroid resistant $\mathrm{T}$ cellmediated rejection with a MELD $\geq 19$ is associated with suboptimal 5-year LT-free survival. ATG use and re-transplant candidacy needs to be re-evaluated in this clinical cohort.

\section{P085 VIRTUAL TRANSPLANT ASSESSMENT IS FEASIBLE AND MAY INCREASE ACCESS TO LIVER TRANSPLANTATION}

${ }^{1}$ Agimol Pradeep*, ${ }^{1}$ Faye Barker, ${ }^{2}$ Katie Ramos, ${ }^{1}$ Wendy Littlejohn, ${ }^{1}$ Oliver Tavabie, ${ }^{1}$ Chris Nicholson, ${ }^{1}$ Krishna Menon, ${ }^{2}$ Matthew Cramp, ${ }^{3}$ Neil McDougall, ${ }^{3} J o h n n y$ Cash, ${ }^{1}$ Varuna Aluvihare. ${ }^{1}$ King's College Hospital, UK; ${ }^{2}$ Plymouth Hospitals NHS Trust, UK; ${ }^{3}$ Royal Victoria Hospital, UK

\subsection{6/gutjnl-2021-BASL.93}

Background It has been demonstrated that patients living further from a transplant centre are less likely to be transplanted in the UK and abroad. King's College Hospital (KCH) has collaborated with teams in Plymouth, North Bristol and Belfast to establish satellite transplant centres (SLTCs) to address this. However, the COVID-19 pandemic threatened to exacerbate this healthcare inequity by reducing travel and stopping patients undergoing transplant assessment at $\mathrm{KCH}$. In response to this, we developed a virtual transplant assessment (VTA) pathway to ensure that patients at SLTCs were not disadvantaged through the pandemic.

Methods Data were retrospectively collected from all patients referred from our SLTCs and discussed at $\mathrm{KCH}$ transplant listing meetings between April 2020 and April 2021. Patients were either assessed face-to-face or virtually depending on the 'discussion in principle' (DIP) MDM between $\mathrm{KCH}$ and the corresponding SLTC. Demographic (age and sex), clinical (disease aetiology, smoking history, time of referral, time of full discussion and whether the patient was listed for transplant or transplanted) and laboratory data for calculation of prognostic scores were collected from the clinical notes. Continuous variables were analysed for normality using the D'Agostino and Pearson test and patients undergoing face-to-face assessments were directly compared with those undergoing VTA by $t$ test if normally distributed or Mann-Whitney $U$ test if non-normally distributed. Categorical data were analysed using Fisher's exact test. Time from listing to transplantation was compared between both groups by survival analysis.

Results During this time, 19 patients underwent VTA and 30 patients underwent face-to-face assessment. No patients were fully assessed from SLTCs between April and July 2020. 